\title{
TOTAL DUAL INTEGRALITY OF MATCHING FOREST CONSTRAINTS
}

\author{
ALEXANDER SCHRIJVER
}

\author{
Received August 31, 1998
}

\begin{abstract}
Let $G=(V, E, A)$ be a mixed graph. That is, $(V, E)$ is an undirected graph and $(V, A)$ is a directed graph.

A matching forest (introduced by R. Giles) is a subset $F$ of $E \cup A$ such that $F$ contains no circuit (in the underlying undirected graph) and such that for each $v \in V$ there is at most one $e \in F$ such that $v$ is head of $e$. (For an undirected edge $e$, both ends of $e$ are called head of e.)

Giles gave a polynomial-time algorithm to find a maximum-weight matching forest, yielding as a by-product a characterization of the inequalities determining the convex hull of the incidence vectors of the matching forests.

We prove that these inequalities form a totally dual integral system. It is equivalent to an "all-integer" min-max relation for the maximum weight of a matching forest. Our proof is based on an exchange property for matching forests, and implies Giles' characterization.
\end{abstract}

\section{Introduction}

In an interesting trilogy, Rick Giles [9], [10], [11] introduced the concept of a matching forest in a mixed graph - a common generalization of matchings in undirected graphs and branchings in directed graphs - and he gave a polynomial-time algorithm to find a maximum-weight matching forest, yielding as a by-product a characterization of the matching forest polytope (the convex hull of the incidence vectors of matching forests).

Giles' results generalize the polynomial-time solvability and the polyhedral characterizations for matchings (Edmonds [4]) and for branchings (Chu and Liu [2], Edmonds [5], Bock [1]).

Mathematics Subject Classification (1991): 05C70, 90C27, 90C57 
In this paper we prove that the system given by Giles is totally dual integral (cf. [15]). This means that the linear program of maximizing an integer objective function over the constraints has integer primal and dual solutions. It generalizes the total dual integrality of the matching constraints in an undirected graph, proved by Cunningham and Marsh [3] (which generalizes the Tutte-Berge formula for the maximum size of a matching (cf. [13])), and the total dual integrality of the branching constraints in a directed graph, proved implicitly by Edmonds [5], Bock [1], and Fulkerson [8].

We give some definitions and background, and formulate Giles' inequalities for the matching forest polytope.

A mixed graph is a triple $(V, E, A)$, where $(V, E)$ is an undirected graph and $(V, A)$ is a directed graph. (So $E$ and $A$ are disjoint.) In this paper, a graph can have multiple edges, but no loops. If an edge $e$ is directed from $u$ to $v$, then $u$ is called the tail and $v$ the head of $e$. If $e$ is undirected and connects $u$ and $v$, then both $u$ and $v$ are called head of $e$. The underlying undirected graph of a mixed graph is the undirected graph obtained from the mixed graph by forgetting the orientations of the directed edges.

A subset $F$ of $E \cup A$ is called a matching forest if $F$ does not contain any circuit (in the underlying undirected graph) and any vertex $v$ is head of at most one edge in $F$. We call a vertex $v$ a root of $F$ if $v$ is not the head of any edge in $F$. We denote the set of roots of $F$ by $R(F)$.

It is convenient to consider the relations of matching forests with matchings in undirected graphs and branchings in directed graphs.

A matching in an undirected graph $(V, E)$ is a set $M$ of pairwise disjoint edges. So $M$ is a matching if and only if $M$ is a matching forest in the mixed graph $(V, E, \emptyset)$. In this case, the roots of $M$ are the vertices not covered by $M$.

A branching in a directed graph $(V, A)$ is a set $B$ of edges being a forest in the underlying undirected graph, such that each vertex is head of at most one edge in $B$. So $B$ is a branching if and only if $B$ is a matching forest in the mixed graph $(V, \emptyset, A)$. An arborescence is a branching $B$ with exactly one root. So for any branching $B$, each weak component of the directed graph $(V, B)$ is an arborescence, containing precisely one root of $B$. (A weak component of a mixed graph is a component of the underlying undirected graph.)

In turn, we can characterize matching forests in terms of matchings and branchings: for any mixed graph $(V, E, A)$, a subset $F$ of $E \cup A$ is a matching forest if and only if $F \cap A$ is a branching in $(V, A)$ and $F \cap E$ is a matching in $(V, E)$ such that $F \cap E$ only covers roots of $F \cap A$.

It is useful to observe and remember the following formulas, for any matching forest $F$ in a mixed graph $(V, E, A)$, setting $M:=F \cap E$ and 
$B:=F \cap A:$

$$
R(F)=R(M) \cap R(B) \text { and } \quad V=R(M) \cup R(B) .
$$

In fact, for any matching $M$ in $(V, E)$ and any branching $B$ in $(V, A)$, the set $M \cup B$ is a matching forest if and only if $R(M) \cup R(B)=V$.

The matching forest polytope of a mixed graph $(V, E, A)$ is the convex hull of the incidence vectors of the matching forests. The incidence vector of a subset $F$ of $E \cup A$ is the vector $\chi^{F}$ in $\{0,1\} E \cup A$ satisfying $\chi^{F}(e)=1$ if and only if $e \in F$. So the matching forest polytope is a polytope in $\mathbb{R}^{E \cup A}$.

Giles showed that the matching forest polytope is determined by the following inequalities:
(i) $x(e) \geq 0$
for each $e \in E \cup A$,
(ii) $x\left(\delta^{\text {head }}(v)\right) \leq 1$ for each $v \in V$,
(iii) $x(\gamma(\mathcal{L})) \leq\left\lfloor|\cup \mathcal{L}|-\frac{1}{2}|\mathcal{L}|\right\rfloor$ for each subpartition $\mathcal{L}$ with $|\mathcal{L}|$ odd.

Here we use the following notation and terminology. $\delta^{\text {head }}(v)$ denotes the set of edges with head $v$. A subpartition is a collection of pairwise disjoint nonempty subsets of $V$. As usual, $\cup \mathcal{L}$ denotes the he union of the sets in $\mathcal{L}$. For each subpartition $\mathcal{L}$ we set:

$$
\begin{aligned}
& \gamma(\mathcal{L}):=\text { the set of undirected edges contained in } \cup \mathcal{L} \text { and of } \\
& \text { directed edges contained in any set in } \mathcal{L} .
\end{aligned}
$$

Finally, $\lfloor x\rfloor$ denotes the lower integer part of a real number $x$.

The inequalities (i) and (ii) in (2) are trivially valid for the incidence vector of any matching forest $F$. To see that (iii) is valid, we can assume that $F \subseteq \gamma(\mathcal{L})$ and that $V=\cup \mathcal{L}$. Then $|R(F \cap A)| \geq|\mathcal{L}|$, since each set in $\mathcal{L}$ contains at least one root of $F \cap A$. Moreover, $|F \cap E| \leq\left\lfloor\frac{1}{2}|R(F \cap A)|\right\rfloor$, since $F \cap E$ is a matching on $R(F \cap A)$. As $|F \cap A|=|V|-|R(F \cap A)|$, this gives:

$$
\begin{aligned}
|F| & \left.=|F \cap E|+|F \cap A| \leq\left|\frac{1}{2}\right| R(F \cap A) \mid\right\rfloor+(|V|-|R(F \cap A)|) \\
& =\left\lfloor|V|-\frac{1}{2}|R(F \cap A)|\right\rfloor \leq\left\lfloor|\cup \mathcal{L}|-\frac{1}{2}|\mathcal{L}|\right\rfloor
\end{aligned}
$$

as required.

In this paper we show that system (2) is totally dual integral (a concept introduced by Edmonds and Giles [7]). It means that, for each 'weight' function $w: E \cup A \rightarrow \mathbb{Z}$, the linear program of maximizing $w^{T} x$ over (2) has integer optimum primal and dual solutions. It is equivalent to an "allinteger" min-max formula for the maximum weight of a matching forest (for integer weights). 
Our proof implies Giles' characterization of the matching forest polytope, while we do not make use of the theorem of Edmonds and Giles that the existence of integer optimum dual solutions implies the existence of integer optimum primal solutions.

More precisely, our proof consists of showing that for any integer weight function $w$ on $E \cup A$, there exists a matching forest $F$ and an integer dual solution to the linear program of maximizing $w^{T} x$ over (2), of value equal to the weight of $F$. Since the incidence vector of $F$ satisfies (2), standard polyhedral theory then gives that the vertices of the polytope $P$ determined by (2) are exactly the incidence vectors of the matching forests (as any vertex of $P$ is the unique optimum solution of maximizing $w^{T} x$ over (2), for some integer weight function $w$ ).

The total dual integrality of (2) has as special case a min-max formula for the maximum size of a matching forest in a mixed graph $(V, E, A)$. However, a direct formula for this can be easily derived from the Tutte-Berge formula - see Section 4.

Our proof method in fact is a generalization of the proof in [14] of the result of Cunningham and Marsh [3] that the matching constraints are totally dual integral. The proof in [14] uses ideas of Lovász [12] on the characterization of the matching polytope.

\section{An exchange property of matching forests}

Bases of our proof are an exchange property for branchings, and one for matching forests. The latter generalizes a well-known and trivial exchange property for matchings in an undirected graph, based on considering the union of two matchings.

The proofs use the following lemma, that follows directly from a theorem of Edmonds [6] on edge-disjoint branchings. Here $d^{\text {in }}(K)$ denotes the number of edges entering $K$ (a strong component of a directed graph is an inclusionwise maximal set of vertices inducing a strongly connected subgraph):

Lemma 1. Let $B_{1}$ and $B_{2}$ be branchings partitioning the edge set of a directed graph $D=(V, A)$. Let $R_{1}$ and $R_{2}$ be sets with $R_{1} \cup R_{2}=R\left(B_{1}\right) \cup R\left(B_{2}\right)$ and $R_{1} \cap R_{2}=R\left(B_{1}\right) \cap R\left(B_{2}\right)$. Then $A$ can be split into branchings $B_{1}^{\prime}$ and $B_{2}^{\prime}$ with $R\left(B_{i}^{\prime}\right)=R_{i}$ for $i=1,2$ if and only if each strong component $K$ of $D$ with $d^{\mathrm{in}}(K)=0$ intersects both $R_{1}$ and $R_{2}$.

Proof. Necessity is easy, since the root set of any branching intersects any component $K$ with $d^{\text {in }}(K)=0$. 
To see sufficiency, by the theorem of Edmonds [6], branchings $B_{1}^{\prime}$ and $B_{2}^{\prime}$ as required exist, if and only if for each nonempty subset $U$ of $V$ one has

$$
d^{\text {in }}(U) \geq\left|\left\{i \in\{1,2\} \mid U \cap R_{i}=\emptyset\right\}\right| .
$$

(Actually, Edmonds' theorem gives the existence of disjoint branchings $B_{1}^{\prime}$ and $B_{2}^{\prime}$ with $R\left(B_{i}^{\prime}\right)=R_{i}$ for $i=1,2$. The fact that $B_{1}^{\prime} \cup B_{2}^{\prime}=A$ follows from the fact that $\left|B_{1}^{\prime}\right|+\left|B_{2}^{\prime}\right|=\left|B_{1}\right|+\left|B_{2}\right|$, as $\left|R\left(B_{1}^{\prime}\right)\right|+\left|R\left(B_{2}^{\prime}\right)\right|=\left|R\left(B_{1}\right)\right|+\left|R\left(B_{2}\right)\right|$.)

Suppose that inequality (5) does not hold. Then the RHS is positive. If it is 2 , then $U$ is disjoint from both $R_{1}$ and $R_{2}$, and hence from both $R\left(B_{1}\right)$ and $R\left(B_{2}\right)$ (since $R_{1} \cup R_{2}=R\left(B_{1}\right) \cup R\left(B_{2}\right)$ ), implying that both $B_{1}$ and $B_{2}$ enter $U$, and so $d^{\text {in }}(U) \geq 2$.

So the RHS is 1 , and hence the LHS is 0 . We can assume that $U$ is an inclusionwise minimal set with this property. It implies that $U$ is a strong component of $D$. Then by the condition, $U$ intersects both $R_{1}$ and $R_{2}$, contradicting the fact that the RHS in (5) is 1.

First, this implies the following exchange property for branchings:

Theorem 1. Let $D=(V, A)$ be a directed graph, and let $B_{1}$ and $B_{2}$ be branchings in $D$ partitioning $A$. Let $s$ be a root of $B_{2}$ but not of $B_{1}$, and let $r$ be the root of the arborescence in $B_{1}$ containing $s$. Then $A$ can be partitioned into branchings $B_{1}^{\prime}$ and $B_{2}^{\prime}$ with $R\left(B_{1}^{\prime}\right)=R\left(B_{1}\right) \cup\{s\}$ or $R\left(B_{1}^{\prime}\right)=$ $\left(R\left(B_{1}\right) \backslash\{r\}\right) \cup\{s\}$.

Proof. Let $K$ be the strong component of $D$ containing $s$. If no edge of $D$ enters $K$, then $r \in K$ (as $B_{1}$ contains a directed path from $r$ to $s$ ), and hence $r$ is not a root of $B_{2}$ (as otherwise no arc enters $r$ while $K$ is strongly connected); define $R_{1}:=\left(R\left(B_{1}\right) \backslash\{r\}\right) \cup\{s\}$ and $R_{2}:=\left(R\left(B_{2}\right) \backslash\{s\}\right) \cup\{r\}$. Alternatively, if some edge of $D$ enters $K$, define $R_{1}:=R\left(B_{1}\right) \cup\{s\}$ and $R_{2}:=$ $R\left(B_{2}\right) \backslash\{s\}$. Then the Lemma implies that $A$ can be split into branchings $B_{1}^{\prime}$ and $B_{2}^{\prime}$ with $R\left(B_{i}^{\prime}\right)=R_{i}$ for $i=1,2$.

The lemma is also used to prove the following exchange property for matching forests (which can be generalized (with the methods below) so that it contains Theorem 1 - however, a most general statement seems not very attractive to formulate, and therefore we have restricted ourselves to stating what we need for our present purpose):

Theorem 2. Let $(V, E, A)$ be a mixed graph, and let $F_{1}$ and $F_{2}$ be matching forests partitioning $E \cup A$. Let $s \in R\left(F_{2}\right) \backslash R\left(F_{1}\right)$. Then there exist matching forests $F_{1}^{\prime}$ and $F_{2}^{\prime}$ partitioning $E \cup A$ such that $s \in R\left(F_{1}^{\prime}\right)$ and such that 
(6) either (i) $\left|F_{1}^{\prime}\right|<\left|F_{1}\right|$,

$$
\begin{aligned}
& \text { or (ii) }\left|F_{1}^{\prime}\right|=\left|F_{1}\right| \text { and }\left|R\left(F_{1}^{\prime}\right)\right|>\left|R\left(F_{1}\right)\right|, \\
& \text { or (iii) }\left|F_{1}^{\prime}\right|=\left|F_{1}\right|, R\left(F_{1}^{\prime}\right)=\left(R\left(F_{1}\right) \backslash\{t\}\right) \cup\{s\} \text { for some } \\
& t \in R\left(F_{1}\right) \text {, and }\left|R\left(F_{1}^{\prime} \cap A\right) \cap K\right|=\left|R\left(F_{1} \cap A\right) \cap K\right| \\
& \text { for each strong component } K \text { of the directed graph } \\
&(V, A) \text {. }
\end{aligned}
$$

Proof. Define $M_{i}:=F_{i} \cap E$ and $B_{i}:=F_{i} \cap A$ for $i=1,2$. Let $\mathcal{K}$ be the collection of those strong components $K$ of the directed graph $(V, A)$ such that no edge in $A$ enters $K$. Note that each set in $\mathcal{K}$ intersects both $R\left(B_{1}\right)$ and $R\left(B_{2}\right)$, and that for any vertex $v \in R\left(B_{1}\right) \cap R\left(B_{2}\right)$ one has $\{v\} \in \mathcal{K}$.

So each $K \in \mathcal{K}$ with $|K| \geq 2$, intersects $R\left(B_{1}\right)$ and $R\left(B_{2}\right)$ in disjoint subsets. Hence we can choose for each such $K$

$$
\begin{aligned}
& \text { a pair } e_{K} \subseteq K \text { consisting of a vertex in } R\left(B_{1}\right) \backslash R\left(B_{2}\right) \text { and a } \\
& \text { vertex in } R\left(B_{2}\right) \backslash R\left(B_{1}\right) \text {. }
\end{aligned}
$$

Let $N$ be the set of pairs $e_{K}$ for $K \in \mathcal{K}$ with $|K| \geq 2$. So $N$ is a matching on $V$.

Then the undirected graph $H$ on $V$ with edge set

$$
M_{1} \cup M_{2} \cup N
$$

consists of a number of vertex-disjoint paths and circuits, since any vertex in $R\left(B_{1}\right) \backslash R\left(B_{2}\right)$ is not covered by $M_{2}$, and any vertex in $R\left(B_{2}\right) \backslash R\left(B_{1}\right)$ is not covered by $M_{1}$.

Moreover, $s$ is the beginning vertex of a path component $P$ of $H$ (possibly only consisting of $s$ ). Indeed, $s$ is not covered by $M_{2}$, as $s \in R\left(F_{2}\right)=R\left(M_{2}\right) \cap$ $R\left(B_{2}\right)$. If $s$ is covered by $M_{1}$, then $s \in R\left(B_{1}\right)$, and so $s \in R\left(B_{1}\right) \cap R\left(B_{2}\right)$, implying that $s$ is not covered by $N$.

Let $Y$ be the set of edges in $M_{1} \cup M_{2}$ occurring in $P$, and set

$$
M_{1}^{\prime}:=M_{1} \triangle Y \text { and } M_{2}^{\prime}:=M_{2} \triangle Y
$$

(where $\triangle$ denotes symmetric difference). Since $Y$ is the union of the edge set of some (path) components of the graph $\left(V, M_{1} \cup M_{2}\right)$, we know that $M_{1}^{\prime}$ and $M_{2}^{\prime}$ are matchings again.

Then, obviously, $R\left(M_{1}^{\prime}\right)$ and $R\left(M_{2}^{\prime}\right)$ arise from $R\left(M_{1}\right)$ and $R\left(M_{2}\right)$ by exchanging these sets on $V P(=$ the vertex set of $P$ ); that is:

$$
\begin{aligned}
& R\left(M_{1}^{\prime}\right)=\left(R\left(M_{1}\right) \backslash V P\right) \cup\left(R\left(M_{2}\right) \cap V P\right) \quad \text { and } \\
& R\left(M_{2}^{\prime}\right)=\left(R\left(M_{2}\right) \backslash V P\right) \cup\left(R\left(M_{1}\right) \cap V P\right) .
\end{aligned}
$$


We show that a similar operation can be performed with respect to $B_{1}$ and $B_{2}$; that is, we show that there exist disjoint branchings $B_{1}^{\prime}$ and $B_{2}^{\prime}$ satisfying

$$
\begin{aligned}
& R\left(B_{1}^{\prime}\right)=\left(R\left(B_{1}\right) \backslash V P\right) \cup\left(R\left(B_{2}\right) \cap V P\right) \text { and } \\
& R\left(B_{2}^{\prime}\right)=\left(R\left(B_{2}\right) \backslash V P\right) \cup\left(R\left(B_{1}\right) \cap V P\right) .
\end{aligned}
$$

By the Lemma, it suffices to show that each strong component $K$ of $(V, A)$ with $d^{\text {in }}(K)=0$ intersects both sets in (11). If $|K|=1$ then $K$ is contained in both $R\left(B_{1}\right)$ and $R\left(B_{2}\right)$, and hence in both sets in (11). If $|K| \geq 2$, then $e_{K}$ intersects both $R\left(B_{1}\right)$ and $R\left(B_{2}\right)$. Since $e_{K}$ is either contained in $V P$ or disjoint of $V P, e_{K}$ intersects both sets in (11). Hence, as $e_{K} \subseteq K$, also $K$ intersects both sets in (11). Therefore, branchings $B_{1}^{\prime}$ and $B_{2}^{\prime}$ satisfying (11) exist.

(10) and (11) imply:

$$
F_{1}^{\prime}:=M_{1}^{\prime} \cup B_{1}^{\prime} \quad \text { and } \quad F_{2}^{\prime}:=M_{2}^{\prime} \cup B_{2}^{\prime} \text { are matching forests. }
$$

To see this, we must show that $R\left(M_{1}^{\prime}\right) \cup R\left(B_{1}^{\prime}\right)=V$ and $R\left(M_{2}^{\prime}\right) \cup R\left(B_{2}^{\prime}\right)=V$. Since $R\left(M_{1}\right) \cup R\left(B_{1}\right)=V$ and $R\left(M_{2}\right) \cup R\left(B_{2}\right)=V$, this follows directly from (10) and (11). This shows (12).

Since $R(F)=R(M) \cap R(B)$ for any matching forest $F$ (with $M:=F \cap E$ and $B:=F \cap A),(10)$ and (11) imply that also $R\left(F_{1}^{\prime}\right)$ and $R\left(F_{2}^{\prime}\right)$ arise from $R\left(F_{1}\right)$ and $R\left(F_{2}\right)$ by swapping on $P$; that is:

$$
\begin{aligned}
& R\left(F_{1}^{\prime}\right)=\left(R\left(F_{1}\right) \backslash V P\right) \cup\left(R\left(F_{2}\right) \cap V P\right) \text { and } \\
& R\left(F_{2}^{\prime}\right)=\left(R\left(F_{2}\right) \backslash V P\right) \cup\left(R\left(F_{1}\right) \cap V P\right) .
\end{aligned}
$$

This implies:

$$
s \in R\left(F_{1}^{\prime}\right) \backslash R\left(F_{2}^{\prime}\right)
$$

since $s \in V P$ and $s \in R\left(F_{2}\right) \backslash R\left(F_{1}\right)$.

We study the effects of the exchanges (10) and (11), to show that one of the alternatives (6) holds. It is based on the following observations on the sizes of $M_{1}^{\prime}$ and $B_{1}^{\prime}$. Let $t$ be the last vertex of $P$ (possibly $t=s$ ).

Suppose that none of the alternatives (6) holds. If $s=t$, then $s$ is not covered by $M_{1}$, and so $M_{1}^{\prime}=M_{1}$ and $R\left(B_{1}^{\prime}\right)=R\left(B_{1}\right) \cup\{s\}$, implying $\left|F_{1}^{\prime}\right|<$ $\left|F_{1}\right|$, which is alternative (6)(i). So $s \neq t$.

By the exchanges we made, $\left|M_{1}\right|-\left|M_{1}^{\prime}\right|=\left|M_{1} \cap E P\right|-\left|M_{2} \cap E P\right|$ (where $E P=$ the edge set of $P$ ), and $\left|R\left(F_{1}\right)\right|-\left|R\left(F_{1}^{\prime}\right)\right|=\left|R\left(F_{1}\right) \cap V P\right|-\left|R\left(F_{2}\right) \cap V P\right|$. This gives, as alternative (6)(i) does not hold:

$$
\begin{aligned}
& \left|M_{1} \cap E P\right|-\left|M_{2} \cap E P\right|+\left|R\left(F_{1}\right) \cap V P\right|-\left|R\left(F_{2}\right) \cap V P\right| \\
= & \left|M_{1}\right|+\left|R\left(F_{1}\right)\right|-\left|M_{1}^{\prime}\right|-\left|R\left(F_{1}^{\prime}\right)\right|=\left|F_{1}^{\prime}\right|-\left|F_{1}\right| \geq 0 .
\end{aligned}
$$


(The last equality follows from the fact that $\left|F_{i}^{\prime}\right|=|V|-\left|M_{i}^{\prime}\right|-\left|R\left(F_{i}^{\prime}\right)\right|$ for $i=1,2$, since $\left|F_{i}^{\prime}\right|+\left|M_{i}^{\prime}\right|$ is the number of heads of edges in $F_{i}^{\prime}$.)

We next note:

(16) any intermediate vertex $v$ of $P$ does not belong to $R\left(F_{1}\right) \cup R\left(F_{2}\right)$.

For suppose that $v \in R\left(F_{1}\right)$. Then (as $v$ is an intermediate vertex of $P$ ) $v$ is covered by $M_{2}$ and some $e_{K} \in N$. Hence $v \in R\left(B_{2}\right)$, and therefore $v \notin R\left(B_{1}\right)$ (by (7)), contradicting the fact that $v \in R\left(F_{1}\right)$. One similarly shows that $v \notin R\left(F_{2}\right)$, proving (16).

As $s \in R\left(F_{2}\right) \backslash R\left(F_{1}\right)$, (16) implies that

(17) $\left|R\left(F_{1}\right) \cap V P\right| \leq\left|R\left(F_{2}\right) \cap V P\right|$, with equality only if $t \in R\left(F_{1}\right) \backslash R\left(F_{2}\right)$.

With (15) this gives that $\left|M_{1} \cap E P\right| \geq\left|M_{2} \cap E P\right|$.

Let $k$ be the number of edges in $M_{1} \cup M_{2}$ on $P$. Note that the edges in $M_{1} \cup M_{2}$ occur along $P$ alternatingly in $M_{1}$ and $M_{2}$, as any intermediate $e_{K} \in N$ on $P$ connects an edge in $M_{1}$ and an edge in $M_{2}$ (as by (7), $e_{K} \in N$ consists of a vertex not in $R\left(B_{2}\right)$ and a vertex not in $R\left(B_{1}\right)$ ).

Suppose $k$ is odd. Then $\left|M_{1} \cap E P\right|=\left|M_{2} \cap E P\right|+1$. So the last edge in $M_{1} \cup M_{2}$ along $P$ (seen from $s$ ) belongs to $M_{1}$. Moreover, one has that $t \notin R\left(F_{1}\right)$. For if $t \in R\left(F_{1}\right)$, then $t$ is not covered by $M_{1}$, and hence $t$ belongs to some $e_{K}=\{v, t\} \in N$ with $v$ covered by $M_{1}$. Hence $v \in R\left(B_{1}\right)$, and hence $t \notin R\left(B_{1}\right)$ (by $(7)$ ), contradicting the fact that $t \in R\left(F_{1}\right)$. So (17) implies that $\left|R\left(F_{2}\right) \cap V P\right|>\left|R\left(F_{1}\right) \cap V P\right|$. This implies with (15) that $\left|F_{1}^{\prime}\right|=\left|F_{1}\right|$ (as $\left.\left|M_{1} \cap E P\right|=\left|M_{2} \cap E P\right|+1\right)$, and with (13) that $\left|R\left(F_{1}^{\prime}\right)\right|>\left|R\left(F_{1}\right)\right|$. So (6) (ii) holds, a contradiction.

So $k$ is even, and hence $\left|M_{1} \cap E P\right|=\left|M_{2} \cap E P\right|$, which implies with (13), (15) and (17) that $\left|R\left(F_{1}\right)\right|=\left|R\left(F_{2}\right)\right|$ and $t \in R\left(F_{1}\right) \backslash R\left(F_{2}\right)$. Therefore, $R\left(F_{1}^{\prime}\right)=\left(R\left(F_{1}\right) \backslash\{t\}\right) \cup\{s\}$.

Finally, $\left|R\left(B_{1}^{\prime}\right) \cap K\right|=\left|R\left(B_{1}\right) \cap K\right|$ for each strong component $K$ of $D$. This follows directly (with (11)) from the fact that for any $v \in K \cap V P$ one has either $K=\{v\}$ (if $|K|=1$ ) or $v \in e_{K}$ (if $|K| \geq 2$ ). For suppose that $v \in V P$ is not incident with any $e_{K} \in N$. If $v$ is an intermediate vertex of $P$, then $v$ is covered by $M_{1}$ and $M_{2}$ and hence $v$ belongs to $R\left(B_{1}\right)$ and $R\left(B_{2}\right)$. If $v=s$, then $v \in R\left(F_{2}\right)$ (so $v \in R\left(B_{2}\right)$ ) and $v$ is covered by $M_{1}$, so $v \in R\left(B_{1}\right)$. If $v=t$, then $v \in R\left(F_{1}\right)$ (so $v \in R\left(B_{1}\right)$ ) and $v$ is covered by $M_{2}$, so $v \in R\left(B_{2}\right)$.

\section{Total dual integrality of matching forest constraints}

We show that system (2) is totally dual integral, which is equivalent to the following. 
For any weight function $w: E \cup A \rightarrow \mathbb{Z}$, let $\nu_{w}$ denote the maximum weight of a matching forest. Call a matching forest $F w$-maximal if $w(F)=\nu_{w}$. Let $\Lambda$ be the set of subpartitions $\mathcal{L}$ of $V$ with $|\mathcal{L}|$ odd.

Then the total dual integrality of (2) is equivalent to: for any 'weight' function $w: E \cup A \rightarrow \mathbb{Z}$, there exist $y: V \rightarrow \mathbb{Z}_{+}$and $z: \Lambda \rightarrow \mathbb{Z}_{+}$satisfying

$$
\sum_{v \in V} y(v)+\sum_{\mathcal{L} \in \Lambda} z(\mathcal{L})\left\lfloor|\cup \mathcal{L}|-\frac{1}{2}|\mathcal{L}|\right\rfloor \leq \nu_{w}
$$

and

$$
\sum_{v \in V} y(v) \chi^{\delta^{\text {head }}(v)}+\sum_{\mathcal{L} \in \Lambda} z(\mathcal{L}) \chi^{\gamma(\mathcal{L})} \geq w
$$

Theorem 3. For any mixed graph $(V, E, A)$, system (2) is totally dual integral.

Proof. We must prove that for each mixed graph $(V, E, A)$ and each function $w: E \cup A \rightarrow \mathbb{Z}$, there exist $y, z$ satisfying (18) and (19).

In proving this, we can assume that $w$ is nonnegative. For suppose that $w$ has negative entries, and let $w^{\prime}$ be obtained from $w$ by setting its negative entries to 0 . As $\nu_{w^{\prime}}=\nu_{w}$ and $w^{\prime} \geq w$, any $y, z$ satisfying (18) and (19) with espect to $w^{\prime}$, also satisfy (18) and (19) with respect to $w$.

Suppose the theorem is not true. Choose a counterexample $(V, E, A)$ and $v: E \cup A \rightarrow \mathbb{Z}_{+}$with $|V|+|E \cup A|+\sum_{e \in E \cup A} w(e)$ as small as possible.

Then the underlying undirected graph of $(V, E, A)$ is connected, since therwise one of the components will form a smaller counterexample. Mo'eover, $w(e) \geq 1$ for each edge $e$, since otherwise we can delete $e$ to obtain a imaller counterexample.

Then:

20) for each $v \in V$, there exists a $w$-maximal matching forest $F$ with $v \in R(F)$.

'or suppose that such a matching forest does not exist. For any edge $e$, et $w^{\prime}(e):=w(e)-1$ if $v$ is head of $e$ and $w^{\prime}(e):=w(e)$ otherwise. Then ${ }^{\prime} w^{\prime}=\nu_{w}-1$. By the minimality of $w$, there exist $y, z$ satisfying (18) and (19) vith respect to $w^{\prime}$. Replacing $y(v)$ by $y(v)+1$ we obtain $y, z$ satisfying (18) nd (19) with respect to $w$, contradicting our assumption. This proves (20).

This implies:

21) each weak component of the directed graph $(V, A)$ is strongly connected.

To see this, it suffices to show that each directed edge $e=(u, v)$ is contained I some directed circuit. By (20) there exists a $w$-maximal matching forest 
$F$ with $v \in R(F)$. Then the weak component of $F$ containing $v$ is an arborescence rooted at $v$. As $F$ has maximum-weight, $F \cup\{e\}$ is not a matching forest, and hence $F \cap A$ contains a directed $v-u$ path. This makes a directed circuit containing $e$, and proves (21).

Let $\mathcal{K}$ denote the collection of strong components of $(V, A)$. Define $w^{\prime}(e):=w(e)-1$ for each edge $e$. The remainder of this proof consists of showing that $|\mathcal{K}|$ is odd (so $\mathcal{K} \in \Lambda$ ), and that

$$
\nu_{w} \geq \nu_{w^{\prime}}+\left\lfloor|V|-\frac{1}{2}|\mathcal{K}|\right\rfloor
$$

This is enough, since, by the minimality of $w$, there exist $y, z$ satisfying (18) and (19) with respect to $w^{\prime}$. Replacing $z(\mathcal{K})$ by $z(\mathcal{K})+1$ we obtain $y, z$ satisfying $(18)$ and $(19)$ with respect to $w$ (note that $\gamma(\mathcal{K})=E \cup A$ ), contradicting our assumption.

To show (22), choose a $w^{\prime}$-maximal matching forest $F$ of maximum size $|F|$. Under this condition, choose $F$ such that it maximizes $|R(F)|$.

We show that for each $s \in V$ the following holds, where $r$ is the root of the arborescence in $F \cap A$ containing $s$ :

(23) there exist a $t \in R(F)$ and a $w^{\prime}$-maximal matching forest $F^{\prime}$ satisfying $\left|F^{\prime}\right|=|F|, R\left(F^{\prime}\right)=(R(F) \backslash\{t\}) \cup\{s\}$, and $\left|R\left(F^{\prime} \cap A\right) \cap K\right|=$ $|R(F \cap A) \cap K|$ for each strong component $K$ of $(V, A)$; if $r \in R(F)$, then moreover $t=r$, and $R\left(F^{\prime} \cap A\right)=(R(F \cap A) \backslash\{r\}) \cup\{s\}$.

Let $F_{1}:=F$, and let $F_{2}$ be a $w$-maximal forest with $s \in R\left(F_{2}\right)$ (which exists by $(20))$.

If $r \notin R(F)$, apply Theorem 2 to $F_{1}$ and $F_{2}$, in the mixed graph obtained from $(V, E, A)$ by deleting any edge not in $F_{1} \cup F_{2}$, and by replacing any edge in $F_{1} \cap F_{2}$ by two parallel edges. It yields the matching forests $F_{1}^{\prime}$ and $F_{2}^{\prime}$.

If $r \in R(F)$, apply Theorem 1 to $B_{1}:=F_{1} \cap A$ and $B_{2}:=F_{2} \cap A$, in the directed graph obtained from $(V, A)$ by deleting any edge not in $B_{1} \cup B_{2}$, and by replacing any edge in $B_{1} \cap B_{2}$ by two parallel edges. It yields the branchings $B_{1}^{\prime}$ and $B_{2}^{\prime}$ in $(V, A)$ satisfying $R\left(B_{1}^{\prime}\right)=R\left(B_{1}\right) \cup\{s\}$ or $R\left(B_{1}^{\prime}\right)=\left(R\left(B_{1}\right) \backslash\{r\}\right) \cup$ $\{s\}$. This implies $R\left(B_{2}^{\prime}\right)=R\left(B_{2}\right) \backslash\{s\}$ or $R\left(B_{2}^{\prime}\right)=\left(R\left(B_{2}\right) \backslash\{s\}\right) \cup\{r\}$. Now define $F_{i}^{\prime}:=\left(F_{i} \cap E\right) \cup B_{i}^{\prime}$ for $i=1,2$. Then the $F_{i}^{\prime}$ are matching forests, since $r \in R\left(F_{1} \cap E\right)$ and $s \in R\left(F_{2} \cap E\right)$.

Note that in both constructions, $\left|F_{1}^{\prime}\right| \leq\left|F_{1}\right|$, and if $\left|F_{1}^{\prime}\right|=\left|F_{1}\right|$ then $\left|R\left(F_{1}^{\prime}\right)\right| \geq\left|R\left(F_{1}\right)\right|$. Moreover,

$$
\chi^{F_{1}^{\prime}}+\chi^{F_{2}^{\prime}}=\chi^{F_{1}}+\chi^{F_{2}}
$$


which implies that $w\left(F_{1}^{\prime}\right)+w\left(F_{2}^{\prime}\right)=w\left(F_{1}\right)+w\left(F_{2}\right)$. Hence

$$
\begin{aligned}
w^{\prime}\left(F_{1}^{\prime}\right)+w\left(F_{2}^{\prime}\right) & =w\left(F_{1}^{\prime}\right)+w\left(F_{2}^{\prime}\right)-\left|F_{1}^{\prime}\right| \geq w\left(F_{1}\right)+w\left(F_{2}\right)-\left|F_{1}\right| \\
& =w^{\prime}\left(F_{1}\right)+w\left(F_{2}\right) .
\end{aligned}
$$

Therefore, since $F_{1}$ is a $w^{\prime}$-maximal matching forest and $F_{2}$ is a $w$-maximal matching forest, we have equality throughout in (25). So $F_{1}^{\prime}$ is $w^{\prime}$-maximal and $\left|F_{1}^{\prime}\right|=\left|F_{1}\right|$. Hence $\left|R\left(F_{1}^{\prime}\right)\right| \geq\left|R\left(F_{1}\right)\right|$. Then, by the maximality of $|R(F)|$, we know that $\left|R\left(F_{1}^{\prime}\right)\right|=\left|R\left(F_{1}\right)\right|$. Therefore, setting $F^{\prime}:=F_{1}^{\prime}$, the conclusions in Theorems 2 and 1 give (23).

Note that (23) implies in particular that $R(F) \neq \emptyset$. Suppose $|R(F)| \geq 2$. Choose $F$ under the additional condition that the minimum distance in $(V, E, A)$ between distinct vertices $u, v \in R(F)$ is as small as possible. Here, the distance in $(V, E, A)$ is the length of a shortest $u-v$ path in the underlying undirected graph.

Necessarily, this distance is at least two, since otherwise we can extend $F$ by an edge connecting $u$ and $v$, thereby maintaining $w^{\prime}$-maximality but increasing the size. This contradicts the maximality of $|F|$.

So we can choose an intermediate vertex $s$ on a shortest $u-v$ path. Let $F^{\prime}$ be the matching forest described in (23), with $t \in R(F)$. By symmetry of $u$ and $v$ we can assume that $t \neq u$. Then $u, s \in R\left(F^{\prime}\right)$, contradicting the choice of $F$, as the distance of $u$ and $s$ is smaller than that of $u$ and $v$.

This implies that $|R(F)|=1$. Let $R(F)=\{r\}$, and let $K$ be the strong component of $(V, A)$ containing $r$. We choose $F$ under the additional constraint that $|R(F \cap A) \cap K|$ is as large as possible.

Suppose $|R(F \cap A) \cap K| \geq 2$. Choose $F$ under the additional constraint that $r$ has minimal distance in $(V, A)$ from some root $u$ of $F \cap A$ in $K \backslash\{r\}$. Here, the distance in $(V, A)$ from $u$ to $r$ is the length of a shortest directed $u-r$ path.

Let $T$ be the arborescence in $F \cap A$ containing $r$. Let $s$ be the first vertex in $T$ on a shortest directed $u-r$ path $Q$ in $(V, A)$. Necessarily $s \neq r$, since otherwise we can extend $F$ by the last edge of $Q$, contradicting the maximality of $|F|$.

Let $F^{\prime}$ be the matching forest described in (23). Then $s \in R\left(F^{\prime}\right)$ and $R\left(F^{\prime} \cap A\right)=(R(F \cap A) \backslash\{r\}) \cup\{s\}$. Hence $u$ remains a root of $F^{\prime} \cap A$, while the distance in $(V, A)$ from $u$ to $s$ is shorter than that from $u$ to $r$. This contradicts our choice of $F$.

So $|R(F \cap A) \cap K|=1$. Suppose that there exists a component $L$ of $(V, A)$ with $|R(F \cap A) \cap L| \geq 2$. Choose $s$ in $L$ arbitrarily. Let $F^{\prime}$ be the matching forest described in (23). Then $s \in R\left(F^{\prime}\right)$ while $\left|R\left(F^{\prime} \cap A\right) \cap L\right| \geq 2$, contradicting the choice of $F$. 
So such a component $L$ does not exist; that is, each $L \in \mathcal{K}$ contains exactly one root of $F \cap A$. So $|F \cap A|=|V|-|\mathcal{K}|$. Moreover, as $|R(F)|=1,|\mathcal{K}|$ is odd and $|F \cap E|=\left\lfloor\frac{1}{2}|\mathcal{K}|\right\rfloor$. So $|F|=|F \cap A|+|F \cap E|=\left\lfloor|V|-\frac{1}{2}|\mathcal{K}|\right\rfloor$. Hence

$$
\nu_{w} \geq w(F)=w^{\prime}(F)+|F|=\nu_{w^{\prime}}+|F|=\nu_{w^{\prime}}+\left\lfloor|V|-\frac{1}{2}|\mathcal{K}|\right\rfloor,
$$

thus proving (22).

We remark that the optimum dual solution $y, z$ constructed in this proof has the following additional property: if $\mathcal{K}, \mathcal{L} \in \Lambda$ and $z(\mathcal{K}), z(\mathcal{L})>0$, then $\mathcal{K}$ and $\mathcal{L}$ are "laminar" in the following sense:

$$
\begin{aligned}
& \forall K \in \mathcal{K} \exists L \in \mathcal{L}: K \subseteq L, \\
& \text { or } \forall L \in \mathcal{L} \exists K \in \mathcal{K}: L \subseteq K, \\
& \text { or } \forall K \in \mathcal{K} \forall L \in \mathcal{L}: K \cap L=\emptyset .
\end{aligned}
$$

\section{The maximum size of a matching forest}

The total dual integrality of (2) has as special case a min-max formula for the maximum size of a matching forest in a mixed graph $(V, E, A)$. However, this min-max formula can be easily derived from the Tutte-Berge formula with the following direct formula.

Let $\mathcal{K}$ be the collection of strong components $K$ of the directed graph $(V, A)$ with the property that no directed edge enters $K$. Consider the undirected graph $H$ with vertex set $\mathcal{K}$, where two distinct $K, L \in \mathcal{K}$ are adjacent if and only if there is an edge in $E$ connecting $K$ and $L$.

Then one has:

(28) The maximum size of a matching forest in $(V, E, A)$ is equal to $\nu(H)+|V|-|\mathcal{K}|$,

where $\nu(H)$ denotes the maximum size of a matching in $H$.

To see (28), let $M^{\prime}$ be a maximum-size matching in $H$. Then $M^{\prime}$ yields a matching $M$ in $(V, E)$. Now there exists a branching $B$ in $(V, A)$ with the property that $B$ has exactly $|\mathcal{K}|$ roots, such that each $K \in \mathcal{K}$ contains exactly one root and such that each vertex covered by $M$ is a root of $B$. (To see that such a branching $B$ exists, choose, for any $K \in \mathcal{K}$ not intersecting $M$, an arbitrary vertex in $K$. Let $X$ be the set of chosen vertices together with the vertices covered by $M$. As $X$ intersects each $K \in \mathcal{K}$, each vertex in $V$ is reachable in $(V, A)$ by a directed path from $X$. Hence there exists a branching $B$ with root set $X$. This $B$ has the required properties.) 
Then $M \cup B$ is a matching forest, of size $\nu(H)+|V|-|\mathcal{K}|$ (as $B$ has size $|V|-|\mathcal{K}|)$.

To see that $M \cup B$ is a maximum-size matching forest, let $F$ be any matching forest. Let $U:=\cup \mathcal{K}$. Then $F$ has at most $|V \backslash U|$ edges with at least one head in $V \backslash U$. Since no directed edge enters $U$, all other edges are contained in $U$. So it suffices to show that $F$ has at most $\nu(H)+|U|-|\mathcal{K}|$ edges contained in $U$.

Let $N$ be the set of (necessarily undirected) edges in $F$ connecting two different components in $\mathcal{K}$. For each $K \in \mathcal{K}$ let $\alpha_{K}$ be the number of edges in $N$ incident with $K$. Then

$$
|N|-\sum_{K \in \mathcal{K}} \max \left\{0, \alpha_{K}-1\right\} \leq \nu(H),
$$

since by deleting, for each $K \in \mathcal{K}$, at most $\max \left\{0, \alpha_{K}-1\right\}$ edges from $N$ incident with $K$, we obtain a matching in the graph $H$ defined above.

We have moreover that any $K \in \mathcal{K}$ contains at most $|K|-\max \left\{1, \alpha_{K}\right\}$ edges of $F$. With (29) this implies that the number of edges in $F$ contained in $U$ is at most

$$
|N|+\sum_{K \in \mathcal{K}}\left(|K|-\max \left\{1, \alpha_{K}\right\}\right)
$$

$\leq \nu(H)+\sum_{K \in \mathcal{K}}\left(\max \left\{0, \alpha_{K}-1\right\}+|K|-\max \left\{1, \alpha_{K}\right\}\right)=\nu(H)+|U|-|\mathcal{K}|$,

as required.

\section{References}

[1] F. Bock: An algorithm to construct a minimum directed spanning tree in a directed network, in: Developments in Operations Research, Volume 1 (Proceedings of the Third Annual Israel Conference on Operations Research, Tel Aviv, 1969; B. AviItzhak, ed.), Gordon and Breach, New York, 1971, 29-44.

[2] Yoeng-Jin ChU, TSENG-HONG LIU: On the shortest arborescence of a directed graph, Scientia Sinica [Peking] 14 (1965), 1396-1400.

[3] W. H. Cunningham, A. B. Marsh, III: A primal algorithm for optimum matching, [in: Polyhedral Combinatorics - Dedicated to the Memory of D. R. Fulkerson (M. L. Balinski, A. J. Hoffman, eds.)] Mathematical Programming Study, 8 (1978), $50-72$.

[4] J. Edmonds: Maximum matching and a polyhedron with 0,1-vertices, Journal of Research National Bureau of Standards Section B, 69 (1965), 125-130. 
[5] J. Edmonds: Optimum branchings, Journal of Research National Bureau of Standards Section B, 71 (1967), 233-240. [reprinted in: Mathematics of the Decision Sciences Part 1 (Proceedings Fifth Summer Seminar on the Mathematics of the Decision Sciences, Stanford, California, 1967; G. B. Dantzig, A. F. Veinott, Jr, eds.) [Lectures in Applied Mathematics Vol. 11], American Mathematical Society, Providence, Rhode Island, 1968, 346-361].

[6] J. Edmonds: Edge-disjoint branchings, in: Combinatorial Algorithms (Courant Computer Science Symposium 9, Monterey, California, 1972; R. Rustin, ed.), Algorithmics Press, New York, 1973, 91-96.

[7] J. Edmonds, R. Giles: A min-max relation for submodular functions on graphs, in: Studies in Integer Programming (Proceedings Workshop on Integer Programming, Bonn, 1975; P. L. Hammer, E. L. Johnson, B. H. Korte, G. L. Nemhauser, eds.) [Annals of Discrete Mathematics 1], North-Holland, Amsterdam, 1977, 185-204.

[8] D. R. FulKerson: Packing rooted directed cuts in a weighted directed graph, Mathematical Programming, 6 (1974), 1-13.

[9] R. Giles: Optimum matching forests I: special weights, Mathematical Programming, 22 (1982), 1-11.

[10] R. Giles: Optimum matching forests II: general weights, Mathematical Programming, 22 (1982), 12-38.

[11] R. GILES: Optimum matching forests III: facets of matching forest polyhedra, Mathematical Programming, 22 (1982), 39-51.

[12] L. LovÁsz: Graph theory and integer programming, in: Discrete Optimization (Proceedings Advanced Research Institute on Discrete Optimization and Systems Applications and Discrete Optimization Symposium, Banff, Alta, and Vancouver, B.C., Canada, 1977; P. L. Hammer, E. L. Johnson, B. H. Korte, eds.) [Annals of Discrete Mathematics 4], North-Holland, Amsterdam, 1979, 141-158.

[13] L. Lovász, M. D. Plummer: Matching Theory, Akadémiai Kiadó, Budapest [also: North-Holland Mathematics Studies Volume 121, North-Holland, Amsterdam], 1986.

[14] A. SCHRIJver: Min-max results in combinatorial optimization, in: Mathematical Programming - The State of the Art (Bonn, 1982; A. Bachem, M. Grötschel, B. Korte, eds.), Springer, Berlin, 1983, 439-500.

[15] A. SCHRIJver: Theory of Linear and Integer Programming, Wiley, Chichester, 1986.

\author{
Alexander Schrijver \\ CWI, Kruislaan 413, \\ 1098 SJ Amsterdam, \\ The Netherlands, \\ and \\ Department of Mathematics, \\ University of Amsterdam, \\ Plantage Muidergracht 24, \\ 1018 TV Amsterdam, \\ The Netherlands \\ Lex.Schrijver@cwi.nl
}

